\title{
EXTREME AVALANCHE RUN-OUT: DATA FROM FOUR MOUNTAIN RANGES
}

\author{
by
}

\author{
D.M. McClung,
}

(Institute for Research in Construction, National Research Council of Canada, 3650 Wesbrook Mall, Vancouver, British Columbia V6S 2L2, Canada)

A.I. Mears,

(Natural Hazards Consultants, 222 East Gothic Avenue, Gunnison, CO 81230, U.S.A.)

and

P. Schaerer

(Institute for Research in Construction, National Research Council of Canada, 3650 Wesbrook Mall, Vancouver, British Columbia V6S 2L2, Canada)

\section{ABSTRACT}

Avalanche run-out distances have a prominent statistical character which is not usually featured in calculations derived from avalanche dynamics models. In order to predict run-out distances in a rational manner, the statistical element should be included. Here, we present and analyze data on extreme run-out from four mountain ranges. When the data are fitted to extreme-value distributions, we are able to show how (and why) extreme-value parameters vary with terrain properties of the different ranges.

\section{INTRODUCTION}

When decisions must be made about definition of safe areas and placement of structures in snow-avalanche terrain, estimation of avalanche-stopping positions is the primary consideration. Speed measurements show that large avalanches slow down very rapidly, and, therefore, use of defence structures will only be possible near the end of the avalanche path. Given all the uncertainties about avalanche velocities, decelerations, and impact pressures, the best policy is to avoid threatened areas.

In spite of the need for high precision in defining safe areas, data show that run-out distances have a statistical character. When reliable long-term data are unavailable or discontinuous, we feel that at present statistical prediction (based upon terrain features) represents the only rational quantitative method for determining run-out distances. The alternative (traditional) approach is based upon attempts to specify friction coefficients in an avalanche-dynamics model to calculate run-out. Uncertainties about flowing snow and its interaction with the terrain make this method highly speculative.

The pioneering efforts in statistical run-out prediction (Bovis and Mears, 1976; Lied and Bakkehöi, 1980; Bakkehöi and others, 1983) were based on regression analyses of topographic parameters. However, McClung and Lied (1987) analyzed data from western Norway and concluded that the data obey a Gumbel (extreme value) distribution. We believe this approach is superior and we have employed it here.

In this paper, we present and analyze extreme run-out distance data from three more mountain ranges: Purcell and Rocky Mountains of Canada, Sierra Nevada of California, and the Colorado Rocky Mountains. These new data are compared with data from western Norway. When all the data are fitted to extreme-value distributions, we are able to show how (and why) the extreme-value parameters vary with the terrain properties of a given range. The variations in predictions also yield the important result that reliable predictions in a given mountain range must be based on a data set for that range.

\section{DESCRIPTION OF DATA}

The four data sets analyzed in this paper consist of "100 year" avalanches. The extreme position reached by avalanche debris was deduced by vegetation damage or the historical record. Although we have attempted to select a 100 year return period, the true return period probably ranges from 50 to 300 years in the data, thus introducing an unavoidable random error into the analysis. This error should diminish in importance as the sample size (number of avalanche paths) increases for a given mountain range.

The data for each mountain range consist of three angles $(\alpha, \beta, \delta)$ and three lengths $\left(H, \Delta x, X_{\beta}\right)$ (Fig. 1). The angle $\alpha$ is defined by sighting from the extreme point reached by avalanches in the past (extreme run-out position) to the starting point; $\beta$ is obtained by sighting from the position where the slope angle first declines to $10^{\circ}$ from the starting point; $\delta$ is the angle defined by sighting between the positions marked for $\alpha$ and $\beta$.

We have chosen the position defined by $\beta$ (the $\beta$ point) as a reference point from which to calculate run-out. The run-out distance $(\Delta x)$ is the horizontal reach from the $\beta$ point to the extreme run-out position. The length $\left(X_{\beta}\right)$ is the horizontal distance between the starting point and the $\beta$ point, and $H$ is the total vertical displacement from the starting point to the extreme run-out position. (Fig. 1)

We have also defined a dimensionless run-out ratio

$$
\frac{\Delta x}{X_{\beta}}=\frac{\tan \beta-\tan \alpha}{\tan \alpha-\tan \delta} .
$$

A total of 397 avalanche paths from the four ranges were included in the analysis: (1) western Norway (127); (2) Rocky Mountains and Purcell Mountains, Canada (125); (3) Rocky Mountains, Colorado (98); (4) Sierra Nevada, 


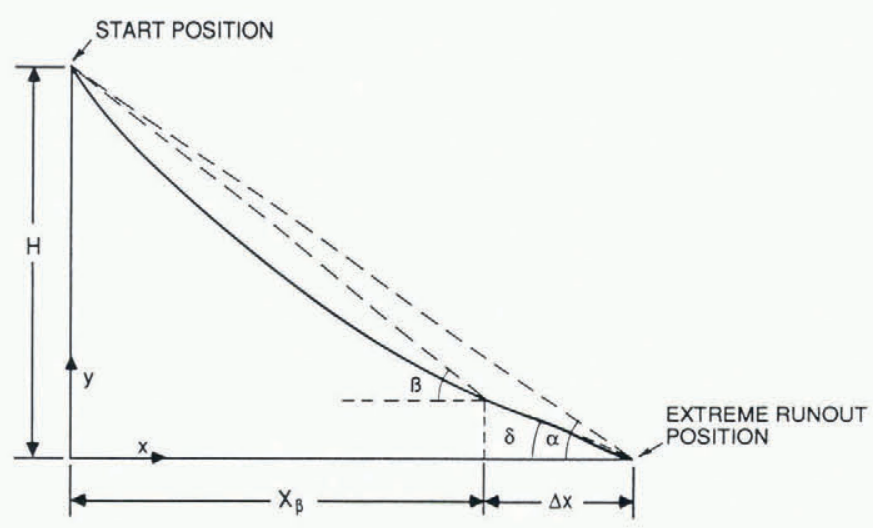

Fig. 1. Geometry and definition of angles and distances.

California (47). Two of these ranges (western Norway and Sierra Nevada) are in maritime climate areas, and the other two are considered continental.

The data-collection methods employed were slightly different for the different ranges, and for completeness we have included the procedures for each below:

(1) Western Norway (McClung and Lied, 1987). The angles $(B, \delta)$ were determined by field measurements or high-quality maps, and $\alpha$ was measured in the field using a clinometer. Extreme run-out positions were determined from long-term historical records. Values for $H$ were calculated by fitting a curve, $y=a x^{2}+b x+c$, to the terrain profile. The value of $y^{\prime}$ at $y=0$ was substracted from the height of the start position to give $H$. From Equation (1), approximate values of $\Delta x$ were calculated using values of $H$ and simple trigonometry.

(2) Rocky and Purcell Mountains, Canada. The angles ( $\alpha$, $\beta, \delta$ ) were measured in the field using a clinometer. Values for $\Delta x$ were measured in the field using a series of distance and angle measurements. The lengths $H$ were estimated from maps by assuming a start position and an extreme run-out position (observed in the field). Vegetative damage (mature timber, approximately 100 years old or older) was used to determine the extreme run-out position.
(3) Rocky Mountains, Colorado, and Sierra Nevada, California. The extreme run-out positions were observed in the field as in Canada. The angles $(\alpha, \beta, \delta)$ were measured in the field on the shorter paths and determined from maps on the longer ones. The values for $H$ and $X_{\beta}$ were scaled from maps, and $\Delta x$ was determined from maps or measured on the ground.

We believe that the values for the angles are within $\pm 0.5^{\circ}$ both for estimates from maps and for those measured in the field. Values of $\Delta x$ are within $30 \mathrm{~m}$ when determined from maps, and within $20 \mathrm{~m}$ from field measurements. Assuming the start positions were correctly identified, $H$ is within about $30 \mathrm{~m}$ when determined from maps. In the present analysis, we have taken only paths for which $H>$ $340 \mathrm{~m}$ in an attempt to minimize the effects of avalanche-deposit length scale on the run-out ratio. We regard the center of mass-stop position as more fundamental than the position of extreme reach but we have no information about the former for our data sets. The extreme run-out position is the quantity of most interest in engineering aspects of zoning.

\section{CHARACTERISTICS OF DATA: DESCRIPTIVE STATIS- TICS}

The mean, standard deviation, and range for all variables used in the analyses are listed in Table I.

Differences in the terrain of the mountain ranges are shown by the values of $\bar{\alpha}$ (the bar denotes a mean value), $\bar{\beta}$, and $\bar{\delta}$. The steepest set of profiles is that from western Norway $\left(\bar{\alpha}=29.4^{\circ}, \quad \bar{\beta}=32.6^{\circ}, \quad \bar{\delta}=6.4^{\circ}\right)$, while the gentlest are from Sierra Nevada $\bar{\alpha}=20.7^{\circ}, \bar{\beta}=26.1^{\circ}$, $\left.\bar{\delta}=4.7^{\circ}\right)$. The longest run-out using either $\Delta x$ or $\Delta x / X_{\beta}$ is from the gentle terrain of Sierra Nevada and Colorado Rockies. From Table I, the steeper paths generally produce lower $\Delta x$ and $\Delta x / X_{\beta}$, but this is not always the case. In general, however, the difference between $\bar{\beta}$ and $\bar{\alpha}$ ranks the same as either $\Delta x$ and $\Delta x / X_{\beta}$. The difference between $\bar{B}$ and $\bar{\alpha}$ is from Table I: $2.0^{\circ}$ (Canada); $3.2^{\circ}$ (Norway); $4.8^{\circ}$ (Colorado); $5.4^{\circ}$ (Sierra Nevada), and it can be seen that both the mean values of $\Delta x$ and $\Delta x / X_{\beta}$ rank in that same order.

By centering and scaling the data, we get the following

TABLE I. DESCRIPTIVE STATISTICS FOR RUN-OUT PARAMETERS FOR FOUR MOUNTAIN RANGES

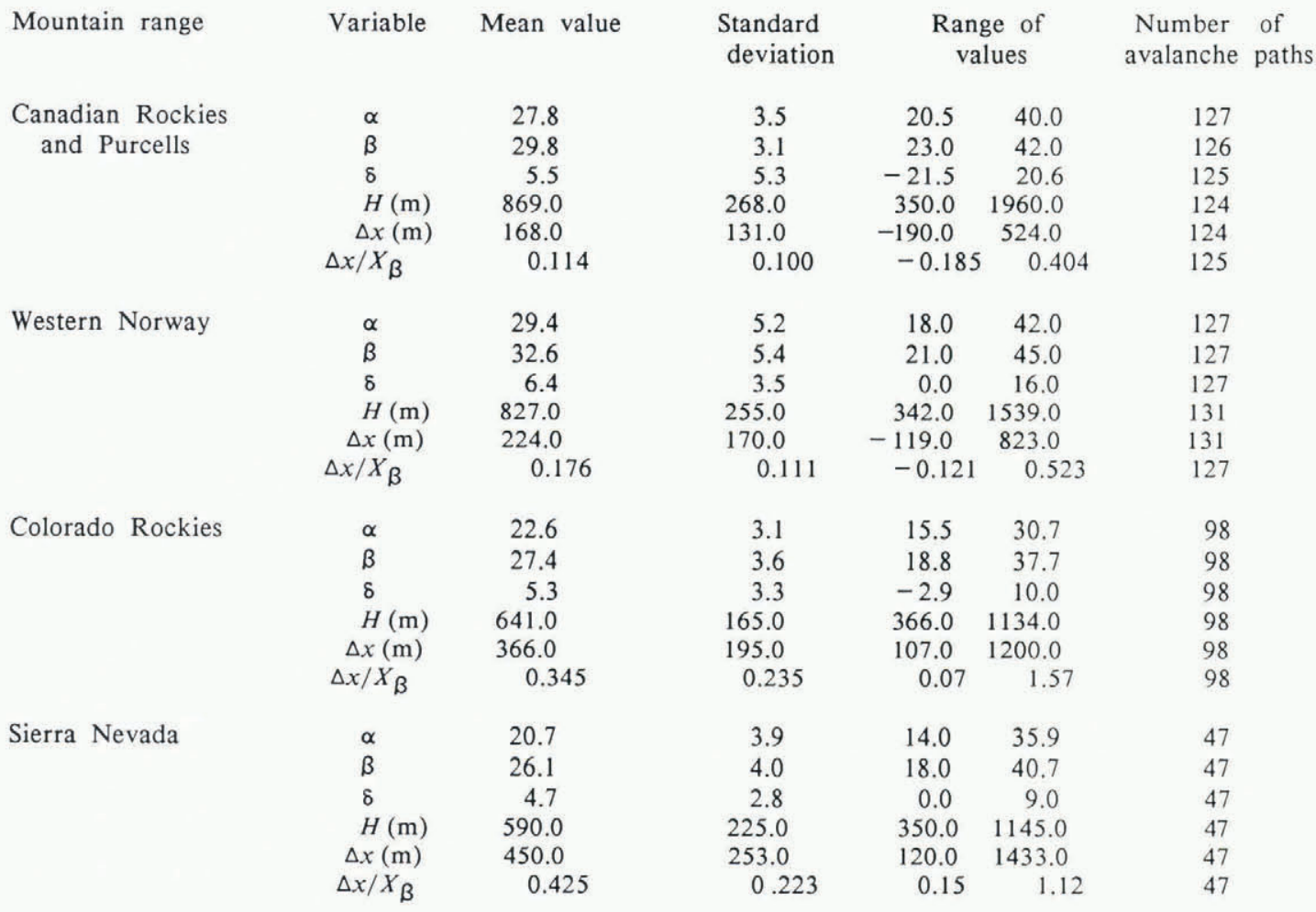


regression equations for the different ranges $(\wedge$ denotes predicted values):

$$
\begin{array}{ll}
\hat{\alpha}=0.93 \beta & \text { Canada }\left(r^{2}=0.75, N=126\right), \\
\hat{\alpha}=0.90 \beta & \text { western Norway }\left(r^{2}=0.87, N=127\right), \\
\hat{\alpha}=0.82 \beta & \text { Colorado }\left(r^{2}=0.60, N=98\right), \\
\hat{\alpha}=0.72 \beta & \text { Sierra Nevada }\left(r^{2}=0.75, N=47\right)
\end{array}
$$

where $r$ is the Pearson (ordinary) correlation coefficient, and $N$ is the number of avalanche paths. When a more general regression equation, $\hat{\alpha}=C_{0} \beta+C_{1}$, is used, the constant $C_{1}$ is not significant except for the data set from Colorado. Therefore, for the other three data sets, Equations (2) represent the best linear regression equations. For example, with the Canadian data, the $t$ statistic for $B$ is 19.1, while for $C_{1}$ it is -0.52 , and calculations with the more general equation show that $C_{1}$ is not significant.

From Equation (1), $\delta$ (or $\bar{\delta}$ ) will also affect the run-out ratio, but it has a smaller effect than the difference between $\beta$ and $\alpha$ illustrated by Equations (2). From Equation (1) and the values in Table I, it can be stated that a large difference between $\beta$ and $\alpha$, as well as a low value of $\alpha$, produces the longest run-out by our definition.

The values in Table I also show that the mean value of run-out (either $\Delta x$ or $\Delta x / X_{\beta}$ ), decreases as the mean value of $H$ increases for some of the data. Since $\Delta x$ and $\Delta x / X_{\beta}$ are not Gaussian variables, rank-correlation coefficients are preferable for determining their relation to other variables. For the individual mountain ranges, the rank correlation coefficients, $r_{S}$, for $\Delta x / X_{\beta}$ correlated with $H$ are: 0.051 (Canada); -0.05 (Norway); -0.41 (Colorado); -0.30 (Sierra Nevada). These results show that, within the data sets, $\Delta x / X_{\beta}$ does not have a significant correlation with $H$ for the data from Canada and Norway, but there is a small negative correlation with $H$ for the data from the U.S.A. We do not have a complete explanation of these results but we note that the terrain is steeper and the vertical relief is higher on average for the data sets from Canada and Norway.

All of the data sets have a non-zero but relatively weak rank correlation for $\Delta x / X_{\beta}$ with $\alpha$ : -0.58 (Canada); -0.39 (Norway); -0.33 (Colorado); -0.51 (Sierra Nevada), but nearly insignificant correlation with $B$ : -0.06 (Canada); -0.07 (Norway); 0.23 (Colorado); 0.04 (Sierra Nevada). If $\beta$ is taken as an index of path steepness, we conclude that the run-out ratio is almost statistically independent of path steepness.

\section{QUANTITATIVE RUN-OUT PREDICTION}

Previously (McClung and Lied, 1987), it was shown that the dimensionless run-out ratio may be fitted to a Gumbel (extreme value) distribution for the data from western Norway. We shall now derive similar predictions for the other data sets. The chosen procedure is to derive approximate estimates of the two parameters for the extreme value distribution: the scale parameter $b$ and the location parameter $u$ by fitting a linear regression equation through plotting positions defined as a function of the nonexcedance probability $p$ for the extreme-value distribution. Let $x \equiv \Delta x / X_{\beta}$, then if $f(x)$ is the probability density function, the non-excedance probability is defined as $p=$ $P_{\mathrm{r}}\left(x \leqslant x_{\mathrm{p}}\right)$ where

$$
p\left(x_{\mathrm{p}}\right)=\int_{-\infty}^{x_{\mathrm{p}}} f(z) \mathrm{d} z=\exp -\exp -\left(\frac{x_{\mathrm{p}}-u}{b}\right) .
$$

Given a value $x_{\mathrm{p}}, 100 \times p \%$ values of $x$ in the distribution have values less than $x_{\mathrm{p}}$. With the size of our data sets (approximately 100 points), values of $p$ of interest range from about 0.5 to 0.99 , corresponding to the median and $1: 100$ run-out ratio, respectively. If run-out distances are found to be much greater than the prediction for $p=0.99$, we conclude that there is a strong probability that such data belong to a different population of values. There are two related reasons why values of $p$ less than 0.5 are not of major interest: (1) in land-use planning, interest is mainly on the extreme values (long-running avalanches), and (2) for data from Norway and Canada the fit to the extreme-value distribution worsens for $p<0.5$, and therefore the accuracy of prediction decreases for the long-running avalanches if values less than 0.5 are included. This is not true for the data from Colorado and Sierra Nevada, but for a consistent comparison of data from all four ranges we have censored all the data in a similar way.

We have defined the non-excedance probability in terms of $N$ values of the run-out ratio, ranked in decreasing value $x_{\mathrm{N}}>x_{\mathrm{N}-1}>x_{1}$ :

$$
p_{i}=\frac{i-0.4}{N} \text {. }
$$

In Equation (4), $i$ is the rank of the observation $x_{i}$. Thus, for the largest run-out ratio $x_{\mathrm{N}}, p_{\mathrm{N}}=(N-0.4) / N$ and for the smallest $p_{i}=0.6 / N$. Equation (4) can be shown to be a good choice for censored extreme-value distributions (paper in preparation by D.M. McClung and A.I. Mears).

We have censored each data set to include values of probability between $\gamma$ and 1 , where $\gamma=0.57721 \ldots$ is Euler's constant. Thus, about $42 \%$ of the highest values for run-out were used in each data set. For the extreme-value distribution, the equation of the model for the regression line through the plotting positions defined by Equation (4) is:

$$
x_{\mathrm{p}}=\left[\frac{\Delta x}{X_{\beta}}\right]_{\mathrm{p}}=u-b \ln (-\ln p)+\varepsilon
$$

where $\varepsilon$ is an error term.

The least-squares procedure above gives values of $b$ and $u$ for each mountain range (the values are listed in Table II). Figure 2 shows the run-out data and regression lines. From Figure 2, the general conclusions from our previous analysis of mean values of run-out are retained. Given a value of $p$, run-out distances generally decrease in the following order: Sierra Nevada, Colorado Rockies, western Norway, Canadian Rockies, and Purcells. The wide variation in extreme-value parameters and run-out clearly shows that it would be unwise to use an equation such as Equation (5) developed from avalanche paths in one mountain range to predict run-out distances in another.

\section{EXTREME-VALUE PARAMETERS AND GEOMETRICAL INTERPRETATION OF RUN-OUT}

For either a full extreme-value distribution or a limited distribution, the scale and location parameters depend only on the mean $\bar{x}$ and standard deviation $\sigma_{x}$ of the extreme values. For a distribution limited at $p=\gamma$, approximate estimates of the scale and location parameters are:

$$
b=0.93 \sigma_{x}
$$

and

$$
u=\bar{x}-1.61 \sigma_{x} .
$$

An approximate value for $\bar{x}=\overline{\left(\Delta x / X_{\beta}\right)}$ may be calculated using Equation (1) with the mean values of $\alpha, \beta$, and $\delta$. For example, with data from Canada, the mean values are: $\bar{\alpha}=25.8^{\circ}, \bar{\beta}=29.4^{\circ}$, and $\bar{\delta}=4.3^{\circ}$, for the extreme values in Figure $2(N=53)$. Calculation using Equation (1) gives $\bar{x}=0.20$, which is the same as the mean of the extreme values. Similar conclusions follow from the other data sets.

From Equations (6) and (7), the scale parameter depends only on the standard deviation of the extreme values, and therefore it characterizes terrain variations within a mountain range. The location parameter depends on both the mean value of the run-out ratio (related to terrain steepness) and the standard deviation (terrain variations) in a mountain range.

Figure 3 illustrates a geometrical interpretation of 
TABLE II. EXTREME VALUE SCALE AND LOCATION PARAMETERS $(b, u)$ FOR FOUR MOUNTAIN RANGES. ALSO GIVEN ARE CORRELATION COEFFICIENT, $r$, AND STANDARD ERRORS, S.E., FOR THE REGRESSION LINES THROUGH THE PLOTTING POSITIONS

Mountain range

Canada

(Rockies, Purcells)

Western Norway

Colorado Rockies

0.118

0.266

Sierra Nevada

0.199

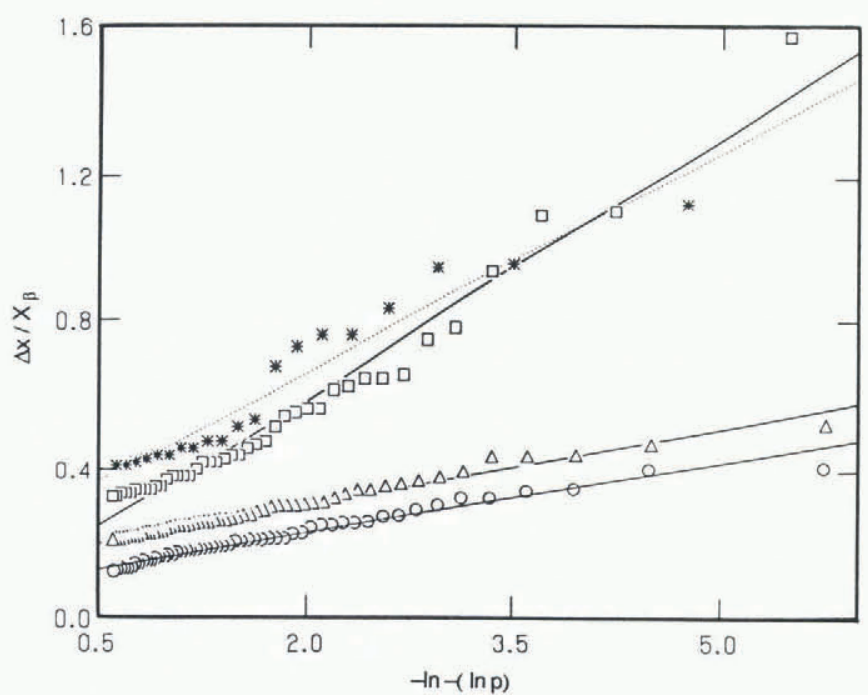

Fig. 2. Plotting positions and regression lines for extremevalue predictions for four mountain ranges. $\left({ }^{*}\right)$ Sierra Nevada, (ㅁ) Colorado Rockies, ( $\Delta$ ) western Norway, (o) Canadian Rockies and Purcells.

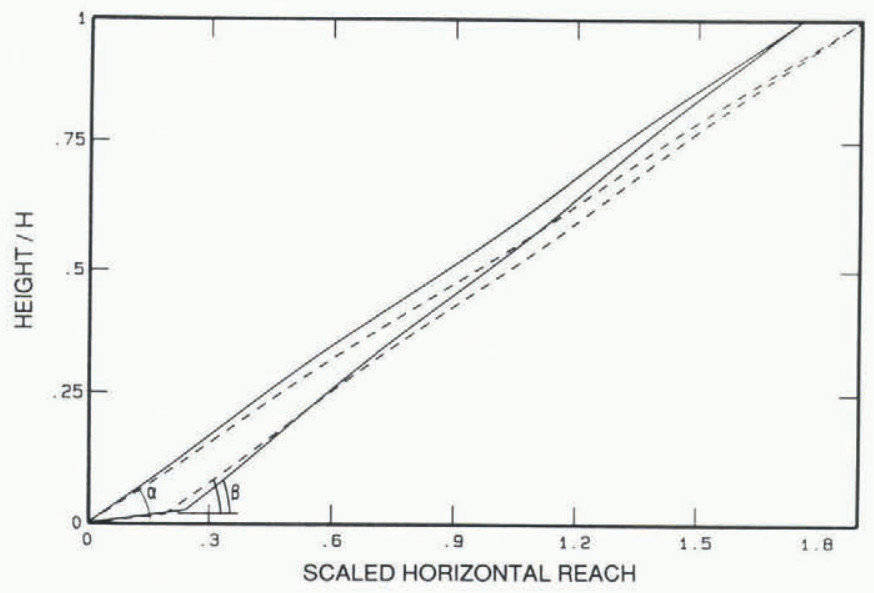

Fig. 3. Geometrical interpretation of run-out ratio using $\bar{\alpha}$, $\bar{\beta}$, and $\bar{\delta}$. (-) western Norway, (---) Canadian Rockies and Purcells.

run-out for data from Norway and Canada. We have used the same vertical drop distances (scaled to unity) with values of $\bar{\alpha}, \bar{\beta}$, and $\bar{\delta}$ from Table $I$. The value of $\Delta x$ is about $1 / 3$ higher for the Norwegian profile in Figure 3, and the values of $\Delta x$ (Table I) differ by this same amount $(33 \%)$. For this particular example, the mean values of $\bar{\alpha}$, $\bar{\beta}$, and $\delta$ are all higher for the Norwegian data than those from Canada. Thus, steeper terrain angles produce higher run-out for the Norwegian data set. This result is, however, the exception rather than the rule. Similar constructions with data from the U.S.A. have shown that the highest mean $r^{2}$

S.E.

Number of

avalanche paths

0.97

0.012

53

0.98

0.010

54

0.97

0.046

42

0.95

0.052

20

run-out (using our definitions) is produced by the greatest differences on average between $\beta$ and $\alpha$ (Equation (2)) combined with the lowest value of $\alpha$. For our data, this combination is fulfilled by the gentlest terrain (Sierra Nevada).

\section{SNOW CLIMATE}

Since our definition of extreme run-out is based on a time-scale of approximately 100 years, we expect that climate regime should not have a strong influence. Experience shows that large dry avalanches have the longest run-out in the majority of cases. Normally, a greater percentage of dry avalanches is expected in a continental climate, with fewer avalanches overall due to smaller amounts of precipitation. We believe that, for time-scales of 100 years, large dry avalanches will occur in either climatic regime to dominate the run-out statistics. By our definitions, the two ranges with the longest run-out distances encompass a maritime climate (Sierra Nevada) and a continental one (Colorado). Similarly, in the case of the shortest run-out, one of the areas is maritime (Norway) and one is continental (Canada). As discussed previously, the tendency for long run-out can be explained by analysis of avalanche-path profiles, but we cannot explain the observed differences through snow-climate classification.

\section{DISCUSSION}

Based on our definitions and analysis, the following conclusions are evident:

There is a significant difference in predicted run-out as terrain in mountain ranges varies. It would not be advisable to predict run-out in one range based on data from another using the methods we have discussed.

If our definition of run-out is accepted, there is no discernible effect based on snow-climate classification. This is expected because of the time-scale our data represent $(\approx 100$ years $)$.

The highest run-out ratio occurs for mountains with the greatest difference between $\beta$ and $\alpha$, and low values for $\alpha$.

We prefer extreme-value prediction of run-out ratio as a measure of run-out rather than the traditional approach based on a regression analysis of $\alpha$ (response variable) as a function of $\beta$ and other terrain variables. In regression analyses with the four data sets, we found that addition of $\delta$ improves predictions of $\alpha$ in a multiple regression analysis with $\beta$ (see McClung and Lied, 1987). However, if $\beta$ is chosen as a reference point, $\delta$ does not qualify as a predictor variable (in general, it would be unknown). Also, no other variables (except $\delta$ ) have been found in combination with $\beta$ to improve predictions of $\alpha$. The run-out ratio $\Delta x / X_{\beta}$ includes the effects of the terrain in the run-out zone ( $\delta$ ) and therefore we feel it is superior.

There is a "scale" effect in some of the data if run-out is defined using the run-out ratio. We have found this in the data from Colorado and Sierra Nevada, but not in data 
from Canada and Norway. Correlation analysis shows that higher vertical drop $H$ tends to produce shorter run-out in Colorado and the Sierra Nevada. With our definition, the mean value of run-out also decreases as the mean value of $H$ increases for individual data sets (Table I). It is possible that the mean value of vertical drop for paths in the two data sets from the U.S.A. is small enough that the scale effect is present. Our attempt to circumvent this problem by choosing paths with $H>340 \mathrm{~m}$ may not have been entirely successful. This question will not be resolved until data on actual avalanche deposits are analyzed to study the difference between statistics for the center of mass-stop position and the position of the extreme tip of the debris.

The run-out ratio is statistically independent of path steepness $(\beta$ or $\tan \beta$ ) for data from Canada, Norway, and the Sierra Nevada, but there is a small positive correlation $\left(r_{\mathrm{S}}=0.23\right)$ for the data from Colorado. The correlations of $\Delta x$ with respect to $\beta$ (or $\tan \beta$ ) are somewhat larger and negative. These results partially justify our preference for the dimensionless run-out ratio, rather than $\Delta x$, as a measure of run-out. Since we are attempting to relate run-out to the non-excedance probability alone, it is preferable that run-out should have minimum correlation with other variables which might qualify as predictors.

We believe that accurate prediction of run-out distances is the most important element in the quantitative engineering aspects of land-use planning in avalanche terrain. We also feel that, when accurate long-term continuous records are not available, prediction based on terrain variables is the only rational method for mathematical prediction so far proposed. Philosophically, our preference would be to specify friction parameters as input to a physically based dynamics model and calculate the speed profile along the incline and the stop position as one problem. This is the traditional approach to the problem (Voellmy, 1955; Perla and others, 1980). With the small amount of information about flowing snow properties available at present, we feel this ultimate goal is somewhat in the future.

In this paper, we have compared data from the four mountain ranges by treating and censoring the data in a consistent manner. None of the equations presented is suitable for actual zoning applications because they are not the optimal equations for the data sets presented.

\section{ACKNOWLEDGEMENTS}

This paper is the product of joint effort involving many years of data collection by (1) the Institute for Research in Construction, NRC Canada; (2) A.I. Mears, Natural Hazards Consultants; (3) the Norwegian Geotechnical Institute. We wish to thank P. Anhorn, B. Benum, and B. Sheffield for their assistance in collection of the Canadian data, and K. Lied for supplying the data from western Norway.

\section{REFERENCES}

Bakkehøi, S., U. Domaas, and K. Lied. 1983. Calculation of snow avalanche runout distance. Ann. Glaciol., 4, 24-29.

Bovis, M.J. and A.I. Mears. 1976. Statistical prediction of snow avalanche runout from terrain variables in Colorado. Arct. Alp. Res., 8(1), 115-120.

Lied, K. and S. Bakkehøi. 1980. Empirical calculations of snow-avalanche runout distance based on topographic parameters. J. Glaciol., 26(94), 165-177.

McClung, D.M. and K. Lied. 1987. Statistical and geometrical definition of snow avalanche runout. Cold Reg. Sci. Technol., 13(2), 107-119.

Perla, R., T.T. Cheng, and D.M. McClung. 1980. A two-parameter model of snow-avalanche motion. $J$. Glaciol., 26(94), 197-207.

Voellmy, A. 1955. Über die Zerstörungskraft von Lawinen. Schweiz. Bauztg, 73(12), 159-162; (15), 212-217; (17), 246-249; (19), 280-285. 